\title{
1 Methods of the Field Work
}

\subsection{Catching}

The point of departure for the standard of all bird ringing station work is the agreedupon number and the quality of catching devices. In most cases, mist-nets are used, and their number and construction determine how to stabilize the catching effort both on a seasonal as well as a long-term scale. If a Heligoland trap is in use, the only standardization problem is the operation time of the trap, which will be influenced by wind force. In modern migration research, the dynamics of seasonal bird migration is the basis for the interpretation of other data. Therefore, stable operating time of Heligoland traps or a standardized number of nets used during the season are an essential methodical requirement. The number of nets in use must be fixed to a level at which the number of available staff can safely handle all birds caught. The main aims of bird ringing station work are: monitoring of bird numbers and seasonal migration dynamics and collecting data for biometrical and other studies of special objectives. Rarely, the work at the ringing station is dedicated only to ring maximum possible numbers of birds.

According to the aims of work at a station, the catching methods must fulfil some requirements (Table 1.1).

Table 1.1: Constrains for different kinds of studies.

\begin{tabular}{ll}
\hline Aim of the study & Number of nets \\
\hline 1. Monitoring & stable within a season, stable between years \\
2. Seasonal dynamics & stable within a season \\
3. Biometrics & recommended stable within a season \\
4. Special studies & recommended stable within a season \\
5. Ringing only & allowed variable number* \\
\hline
\end{tabular}

* but see p. 4 (point 5)

These requirements can be listed more in detail:

\section{Collecting of monitoring data}

In this case, the highest level of standardisation is necessary.

1.1. The work must be planned for a sequence of years.

1.2. Time and period of work is standardized. Within the season, work should be carried out continuously, or at least be made as regular and frequent sampling (this compromise, however, is not recommended!). This is because the migration intensity is very irregular - after a day with no birds, one can have a rush of hundreds or thousands and more birds caught. In the Operation Baltic practice, we had even 
days when around $20 \%$ of yearly catches of one species occurred. Missing such days when sampling could drastically change the total value for the season.

1.3. Equal numbers and quality of nets should be used from year to year, and when new nets are added to standard set, birds caught in the added nets should be treated separately. It must be stressed, however, that any changes affect comparability.

1.3.1. The number of nets should be stable within a season (minor changes may be compensated for when data are evaluated). The nets damaged or stolen should be replaced as soon as possible; good hint: have a stock of a few nets at hand as a backup for replacement.

1.3.2. The daily netting routine should be stable. It is advised to catch birds continuously without closing the nets for night time - in many places, catching peaks do not occur regularly at the same time of the day; e.g. thrushes that have landed after a sea-crossing start to be active in the middle of the day, instead of early in the morning as usual. If possible, do not close nets during migration peaks (unless survival of birds caught is endangered - but also see Laboratory Working Routine hints - p. 118). At some sites, because of special constraints (e.g. high temperatures and insolation in lower latitudes, known and very stable daily catching pattern, and living conditions of the staff), nets may be closed for part of the day. It is advisable to do this regularly at the same time by not prolonging catching because e.g. "there are a lot of birds today", or in order to finish work earlier as it seems a "poor day"). Within Middle East and Northern Africa, a good time for a siesta is between 11.00 and 15.00-16.00.

1.4. Changes of environment must be taken into consideration. Three ways of minimizing the influence from such changes can be listed - (1) arranging the catching area within a relatively stable environment (such habitats are, however, usually not very rich in bird species), (2) controlling growth of trees and bushes (note, however, that the surrounding area will be changing all the time), (3) actively shifting the catching plot within a bigger area of similar environmental conditions (value to birds!). A combination of these methods could be applied according to knowledge of local conditions.

1.5. The nets should be located in different habitats and the distribution of nets relative to habitats ought to be stable over the years.

\section{Seasonal dynamics of migration}

This is one of the most important types of data in any context.

The contents of points 1.2 - 1.3 (above) should be attended to, but any sampling error may affect the picture of seasonal dynamics very much. It is important to remember that during one missing day up to 20 percent of the annual catch of one species could be missed!

\section{Bird measurements}

Catch as many birds as you are able to measure, but note: bird measurements without the possibility to localise these measured birds within their migration waves have very 
limited value! Therefore, adjust number of permanently opened nets to the expected high level of catching (but not to single peaks).

\section{Orientation tests, blood sampling, parasite sampling etc.}

Catch as many birds as you are able to handle with these techniques, but recall previous note under point 3.

\section{Ringing only}

Catch as many birds as you are able to ring (including sex/age determination!). Erect as many nets as you are able to handle without bird losses; eventually use tape-luring, however remember - and once more remember - that station work is not a ringing championship, but means the collecting of scientific data. Today, ringing of migrants is closely connected with collection of other types of data. Seasonal dynamics must be known when ringing recoveries are evaluated in the modern way. Reduce your order for nets, unless they are planned for storage a reserve for replacements, and try to fulfil requirements stated under point 2 .

\subsection{Visual Observations}

Visual observations are frequently performed at ringing stations. They are focused on different groups of species according to the main field of interest of the station staff. Bird counts may be performed in two different ways - (1) counting birds in active migration flights and (2) counting those resting in surroundings of the station area. The first method is used mainly at the "passerine" stations, where both passerines and other diurnal migrants are counted there, as well. The second method is used at "wader" stations, however it is not possible to follow all wader migration as they migrate mostly by night - for more details see Wader Counts - p. 160). In many localities situated at guiding lines like sea coast, spits, and rivers, the stream of diurnal migrants follows a well-defined course, and this may fluctuate within very narrow limits. At other sites, diurnal migration will show "broad-front" character and migrating birds will be dispersed over the whole area. In the first case, the migration count will be more effective, as birds are observed even if actual migration is not intensive. On the other hand, the count could be difficult during a mass passage when there are tens of thousands of migrants per day. Out of concentrated streams of migration, visual observations could be boring, as a low number of migrants are observed, but even in such cases, one could collect interesting data. Areas with no clear guidelines may give biased results due to local changes in the concentration of flying birds, e.g. when parts of flocks may stay outside observers' sight.

Visual observation of the passage should be made from a fixed stand located at the local stream of migration, if there is one within the station area. In order to get good estimation of the total number of birds passing the observation point, all 
day observations should be applied, especially in localities where the intensity of the passage notably varies during the course of a day - this frequently occurs at the sea coast, where some birds have crossed the sea prior to reaching the local stream of migration. Usually, when birds migrate over land, the passage is limited to a few hours after sunrise. In some coastal areas, peaks of diurnal passage occur around noon or even in the afternoon. It is true, however, that observations made during out of the peak of passage are tiresome and boring to the observer, unless they are given a chance to rest. Because of this the observation time during lack of passage can be shortened.

There are two methodical variants of the migration counts used:

1. Continuous observations from sunrise to sunset, or at least for 6-8 hours. Observations are performed on a daily scale throughout the migration period, which is a difficult task, but the result will be the real number of birds passing by an observation point,

2. Sampling observations done on a daily scale and within a day where a sampling procedure is applied (usually 15 minutes per hour) - this method allows estimates of real numbers of birds passing and is not equally exhausting to the observer. Moreover, it does not disturb ringing at the station, because counts are conducted at the time of mist-net checking. The correlation of the results with the first method is at the level 0.90 (after own comparisons of these two methods in early years of the Operation Baltic studies), which can be accepted as very good.

It is critical to stress that, as in catching, any sampling not based on daily counts is biased by the continuously changing migration dynamics of every species, including both diurnal and nocturnal migrants.

The recommended observation routine (Fig. 1.1):

1. The observations of the passage are carried out at 15 min per hour sampling, starting at full hours, and beginning around sunrise and continued till sunset. When there is no observable migration in two consecutive 15 min observations, the next observation is shortened to $5 \mathrm{~min}$; return to the normal routine must be applied consistently when the observer notices intensification of the passage of at least one species. If it is evident after a few years of observation, that the particular locality has no noon and afternoon movements, visual observations might be limited to highly effective times only.

2. At places where intensive bird migration occurs, birds are identified, by sight and sound, and counted within a flexible range - for small birds the range should allow for the identification and count by means of the naked eye, without use of binoculars (when many birds pass, there is no time to check all birds with binoculars); in larger birds (e.g. when raptors are included), the range is limited to a sector within which it is possible to see the bird with the naked eye, but 
where identification is made with the use of binoculars. It is advisable to fix the observation point at such a place, that most of the birds migrating within the local stream pass the observer to the north and west (as they are visible in a better light). As the local stream may shift a little with wind direction and force, it is advisable to shift the observation stand within $100 \mathrm{~m}$ relative to the standard point, adjusting the actual place to better visibility, since the birds passing between the observer and the sun will hardly be identifiable.

3. The birds are noted in a note-book, listing their species name by code - see Species Determination and Coding - p. 81 - in observations (5-letter code is more convenient than the 6-letter one), direction of flight (by wind-rose, 8 directions) and number; birds flying in the most commonly observed direction of the passage (standard direction must be specified at the beginning of the note-book) can be noted as numbers without the direction letters (e.g. CASPI 40, 10, 50...) - all others must be accompanied by the letters describing direction (e.g. SE 30, N $25 . .$.$) , but when non-standard direction is repeated, a bracket could be used (e.g.$ SE [30, 20, 5], E 15...). For standard visual observations, when only total numbers of individuals per species is needed, the subsequent numbers do not describe the size of the flocks passing the observation post, but may be accumulated values for a couple of flocks pooled together. For instance, CASPI 50 does not necessarily mean "a flock of 50 siskins", but could mean: four Siskin flocks: 10, $30,5,5$, adding up to 50 . In this way, notation will be quicker, which is important when a lot of birds migrate. When flock size is wanted, it should be clearly stated in the local instruction.

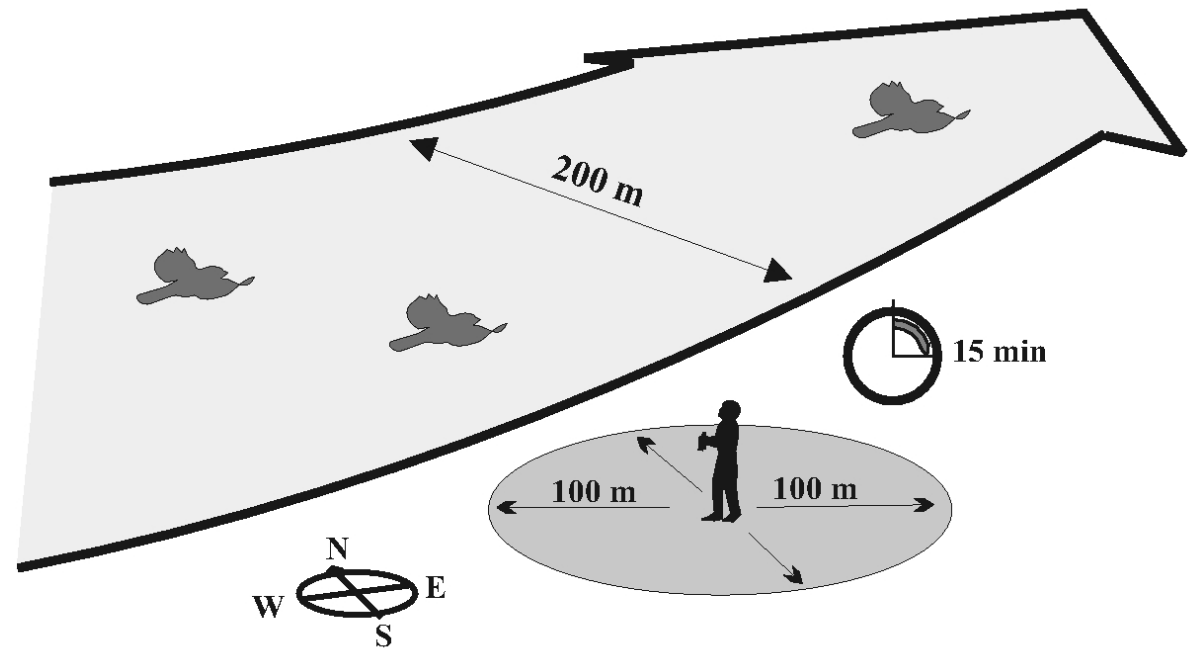

Figure 1.1: Visual observations' routine (see text for explanation). 
A basic rule worth noting is that the same individual should make both observations and notations - there is standardised missing of the birds which pass the point. Noting by dictation to a digital recorder or noting by another person changes detectability of birds and, if applied, it must be used for all observations performed, because of compatibility reasons.

Visual observation of the resting birds is usually performed at the "wader stations" (see Wader Counts - p. 160). 